\title{
Severe Hypokalemia due to Excessive Coca-Cola Consumption, Presenting with Tetraparesis, Rhabdomyolysis, Atrial Fibrillation and Hypokalemic Nephropathy - Case Report
}

\author{
Elena Zakharova* \\ Department of Nephrology, Moscow City Hospital n.a. S.P Botkin
}

Received: July 06, 2016; Accepted: : July 13, 2016; Published: July 22, 2016

*Corresponding author: Elena Zakharova, Department of Nephrology, Moscow City Hospital n.a. S.P Botkin, Tel: +79671346936; Fax: +74999451756; E-mail: helena.zakharova@gmail.com

\begin{abstract}
Potassium deficiency cause cardiovascular, hormonal, muscular hepatic and renal disturbances. Hypokalemia increases the risk for a variety of arrhythmias; impairs skeletal muscle contraction; reduces skeletal blood flow, which can predispose to rhabdomyolysis during exercise; and leads to several disturbances of renal function. The latter include reduced medullary blood flow and increased renal vasculature resistance, which predispose to tubulointerstitial and cystic changes, alterations in acid-base balance and renal concentrating mechanisms. Patients with hypokalemic nephropathy present with nocturia, polyuria and polydipsia, associated with collecting tubule responsiveness to vasopressin. Causes of hypokalemia are multiple and include deficient potassium intake, increased renal potassium losses, increased extrarenal potassium losses, potassium redistribution and pseudohypokalemia, with many nutritional, drug-induced, endocrine and genetic mechanisms. Hypokalemia in persons consuming large amounts of Coca-Cola, first time described in 1994, and since that time reported in a handful of papers, is attributed to the effects of caffeine and fructose, causing the shift of potassium from extracellular space into cells, increased renal excretion of potassium, osmotic diarrhea, osmotic diuresis and inadequate potassium intake. Most papers report myopathy and paralysis, but do not address the issue of renal abnormalities due to Coca-Cola excessive consumption. Here we present a patient, used to consume more than 2 liters of Coca-Cola daily over 20 years, and manifested with the full blown symptoms of severe hypokalemia, which were successfully treated with potassium repletion.
\end{abstract}

Keywords: Hypokalemia causes; Hypokalemic nephropathy

\section{Introduction}

Potassium deficiency cause cardiovascular, hormonal, muscular, hepatic and renal disturbances. Hypokalemia increases the risk for a variety of arrhythmias; impairs skeletal muscle contraction; reduces skeletal blood flow, which can predispose to rhabdomyolysis during exercise; and leads to several disturbances of renal function. The latter include reduced medullary blood flow and increased renal vasculature resistance, which predispose to tubulointerstitial and cystic changes, alterations in acid-base balance and renal concentrating mechanisms. Patients with hypokalemic nephropathy present with nocturia, polyuria and polydipsia, associated with collecting tubule responsiveness to vasopressin. Average duration of hypokalemia, reported in chronic hypokalemic nephropathy is between 3.5 and 9 years [1-6].

Causes of hypokalemia are multiple and may be divided into 5 groups: deficient potassium intake, increased renal potassium losses, increased extrarenal potassium losses, potassium redistribution and pseudohypokalemia [7-11].

1. Decreased intake may result from eating disorders, dental problems, inadequate quantity or quality of food, or potassium-poor total parenteral nutrition.

2. Renal potassium loss is the most common cause of hypokalemia and can occur from medications, endogenous hormone production and, rarely, from intrinsic renal defects.

- Medications include: thiazide and loop diuretics; certain antibiotics, like carbenicillin, amphotericin, aminoglycosides; and cysplatin. Certain herbal products, like licorice and gan cao may be causative for hypokalemia. Osmotic diuresis caused by mannitol usage (or by hyperglycemia) can also lead to potassium renal loss.

- Aldosterone is the most important hormone, causing hypokalemia by stimulating renal potassium excretion and also potassium intake into cells. Excessive aldosterone production may result from congenital adrenal hyperplasia, glucocorticoid treatment, some genetic defects and hyperreninemia.

- Intrinsic renal potassium transport defects are rare and include Bartter syndrome, Gitelman's syndrome and Liddle syndrome. 
3. Non-renal losses include excessive sweating, vomiting, diarrhea and use of laxatives.

4. Potassium redistribution occur due to potassium shifts from extracellular to intracellular compartment, caused by many hormones, particularly insulin, aldosterone, and $\beta_{2}$-adrenergic agonists. It may also be caused by alkalosis or excessive bicarbonate treatment of acidosis. In rare cases hyperparathyroidism or genetic defect of dyhydropiridine-sensitive calcium channels may result in hypokalemic periodic paralysis.

5. Pseudohypokalemia most commonly is caused by acute myeloblastic leukemia, in which abnormal leukocytes take up potassium when blood is stored in the collection vial for a long time at room temperature.

First case of hypokalemia, associated with heavy Coca-Cola consumption, was described in 1994 [12]. Since that time a number of case reports came up, describing patients who drank large volumes of Coca-Cola and developed a severe degree of hypokalemia and paralysis $[13,14]$. The first interpretation of this phenomenon postulated that Coca-Cola contains large amount of caffeine, which enhances the shift of potassium from extracellular space into cells, and increase renal excretion of potassium via renin release, $\beta$-adrenergic stimulation and hyperventilation with alkalosis [13-17]. Other explanations refer to several mechanisms, all of which may play a role simultaneously: Cola contains large amount of fructose, which is absorbed in limited quantities (only about $40 \%$ as compared to glucose) in the small intestine, therefore, unabsorbed fructose pass into the colon, causing an osmotic diarrhea and chronic potassium depletion; elevated glucose levels secondary to excessive cola consumption lead to osmotic diuresis, resulting in the urinary loss of potassium; hyperinsulinemia subsequent to hyperglycemia causes intracellular migration of potassium. Inadequate dietary potassium is supposed to play a role as well - Cola contains small amount of potassium, and as the appetite in persons, constantly consuming Cola, is lowered by fructose intake, lack of balanced diet and lack of consumption of fruits or other dietary ingredients rich in potassium lead to insufficient repletion of potassium, along with excessive potassium losses [17-19]. All these publications describe myopathy and paralysis, but do not address the issue of renal abnormalities.

Here we present a case of severe hypokalemia, admitted to ICU with tetraparesis and diagnosed with muscle, cardiac and renal complications.

\section{Caucasian female, 36 years old, admitted March 01 2016}

Main complains: weakness, inability to move.

Previous medical history: unremarkable, except one pregnancy with caesarian section at 22-23weeks of gestation due to premature placental abruption. Patient denies diabetes, food restriction, usage of antibiotics, diuretics, laxatives and any herbal products. Family history is also unremarkable.
History of present illness: February 222016 after physical exercise she felt low extremities muscle tension and hip muscle pain, which regressed spontaneously in two days. However three days later she developed low grade fever, thirst, dry mouth, progressive muscle weakness and numbness.

Next night she was unable to be up and about, fell of her bed, and for 6 hours lay on the floor without movement. She denies loss of awareness, but reports single episode of vomiting and headache.

February 29shewasadmitted to theinfectious disease hospital, suspected with meningitis. She was conscious, alert, RR 24 per minute, BP 120/70 mm Hg, HR 88 per minute. Neurological exam did not reveal any meningeal signs but confirmed tetraparsesis, work-up showed moderate leukocytosis with normal differential count, thrombocytosis, mild hyperglycemia and high CK level with normal CK-MB (Tables 1,2).Anti-CMV and Anti-EBV IgG positive, anti-CMV and anti-EBV IgM negative. Liquor: colorless, transparent, protein $4.2 \mathrm{~g} / \mathrm{L}$, cytosis $1 / 3$, glucose $4.5 \mathrm{mmol} / \mathrm{L}$. Chest and paranasal sinuses X-Ray unremarkable. The diagnosis of meningitis was ruled out. Patient was suspected with GuillainBarre syndrome and referred to our hospital March 12016.

At admission to ER: Conscious, alert. Body temperature $36.4^{\circ} \mathrm{C}$, RR 18 per minute, pulse regular 90 per minute, $\mathrm{BP} 120 / 80 \mathrm{~mm} \mathrm{Hg}, \mathrm{SpO}_{2} 98 \%$ (room air).Well nourished, skin dry, normally coloured, no oedema or palpable peripheral lymph nodes. HEENT and neck otherwise normal. Lungs: no dullness to percussion, any ronchi, wheezes or rubs. Heart: regular rhythm, no murmur. Abdomen soft, non-tender, bowel sounds normal. Liver $+1 \mathrm{~cm}$ below rib arch, non-painful, spleen and kidneys not felt. Urination is free, urine colourless. Neurologic exam: no meningeal signs; pupils round, D = S, normal photoreaction; tetraparesis, upper limbs - grade 2, lower limbs - grade 1, muscle tone and power decreased; tendon reflexes depressed, D = S; feet signs negative; no dysaesthesia.

Point-of-care work-up in the ER: moderate leukocytosis with normal differential count, severe hypokalemia, CK even higher than before with normal CK-MB, slightly elevated aminotransferase and mild hyperglycemia, other blood chemistry tests within normal range (Tables 1,2). Urinalysis showed low specific gravity and moderate proteinuria and leukocyturia (Table 3).Electrocardiogram: atrial fibrillation with regular rhythm 2:1, 95 bites per minute, QT interval elongation. Brain and spinal MRI did not confirm demyelinating lesions, diagnosis of Guillain-Barre syndrome was not confirmed.

Treatment and further work-up in ICU: Due to severe hypokalemia with atrial fibrillation patient was admitted to ICU and started on intravenous potassium chloride infusions $40 \mathrm{mEq} / \mathrm{h}$, and heparin. Urethral catheter was placed; urine output reached $7000 \mathrm{ml} /$ day. Under treatment in next 4 days her serum potassium gradually elevated to normal range, ECG showed sinus rhythm, muscle weakness partially resolved; urine output became $3500 \mathrm{ml} /$ day. However CK and aminotransferase were still growing, myoglobin was also elevated. Glucose and WBC count became normal, Hb level slightly decreased (Tables 
Severe Hypokalemia due to Excessive Coca-Cola Consumption, Presenting with Tetraparesis, Rhabdomyolysis, Atrial Fibrillation and Hypokalemic Nephropathy - Case Report

Table 1: Blood count data during hospitalisation.

\begin{tabular}{|l|l|l|l|l|}
\hline \multicolumn{2}{|l|}{ Table 1: Blood count data during hospitalisation. } & HB(g/d L) & Plt x 10 $/ \mathbf{L}$ \\
\hline February 29 & WBC $\mathbf{~ 1 0} / \mathbf{L}$ & 15.1 & 517 & 21 \\
\hline March 1 & 20.1 & 13.0 & 279 & 25 \\
\hline March 4 & 25.1 & 9.9 & 314 & 15 \\
\hline March 14 & 9.6 & 13.5 & 263 & 24 \\
\hline
\end{tabular}

Table 2: Blood chemistry data during hospitalisation.

\begin{tabular}{|c|c|c|c|c|}
\hline & February 29 & March 1 & March 4 & March 14 \\
\hline Potassium mmol/ L & & 1.4 & 3.5 & 4.5 \\
\hline CK U/ L & 1836 & 2730 & 31782 & 235 \\
\hline CK-MB U/ L [\%] & $63[3.3]$ & $68.9[2.5]$ & & \\
\hline Al AT U/ L & & 39 & 143 & 16 \\
\hline As AT U/L & & 60 & 342 & 36 \\
\hline Glucose mmol/ L [mg/ dL] & $9.6[172.8]$ & $7.7[138.6]$ & 5.2 [93.6] & $4.6[82.8]$ \\
\hline Urea $\mu \mathrm{mol} / \mathrm{L}$ [mg/ dL] & $6.6[39.9]$ & $6.3[38.1]$ & & $3.7[21.6]$ \\
\hline Creatinine $\mu \mathrm{mol} / \mathrm{L}$ [mg/ dL] & 94 [1.06] & $97[1.2]$ & & $75[0.9]$ \\
\hline Total Bilirubin $\mu \mathrm{mol} / \mathrm{L}$ [mg/ dL] & & $10[0.5]$ & & \\
\hline Total Protein g/ L & & 71.4 & & \\
\hline Lactate $\mathrm{mmol} / \mathrm{L}[\mathrm{mg} / \mathrm{dL}]$ & & $3.5[31.5]$ & $1.2[10.8]$ & \\
\hline Sodium mmol/ L & & 134 & 137 & 138 \\
\hline Chloride mmol/L & & 112 & 115 & 105 \\
\hline Calcium ionized mmol / $\mathrm{L}$ & & 1.31 & 1.20 & \\
\hline Magnesium mmol/L & & 0.74 & & \\
\hline Bicarbonate mmol/L & & 16.1 & 18.4 & \\
\hline pH & & 7.25 & 7.33 & 7.36 \\
\hline Osmolality m0sm/ kg & & 276.7 & 278.2 & \\
\hline Myoglobin $\mu \mathrm{g} / \mathrm{L}$ & & & 1710 & \\
\hline
\end{tabular}

Table 3: Urinalysisdata during hospitalisation.

\begin{tabular}{|l|l|l|l|l|l|l|l|l|l|l|}
\hline & Color & SG & $\mathbf{p H}$ & $\begin{array}{l}\text { Protein } \\
\text { g/ L }\end{array}$ & $\begin{array}{l}\text { Glucose } \\
\text { mmol/ L }\end{array}$ & $\begin{array}{l}\text { WBC } \\
\text { hpf }\end{array}$ & $\begin{array}{l}\text { RBC } \\
\text { hpf }\end{array}$ & $\begin{array}{l}\text { Casts } \\
\text { hpf }\end{array}$ & $\begin{array}{l}\text { Urobilin } \\
\boldsymbol{\mu m o l} / \mathbf{L}\end{array}$ & Crystals \\
\hline March 2 & Light yellow & 1010 & 7.0 & 1.0 & abs & $50-70$ & abs & abs & abs & abs \\
\hline March 14 & Light yellow & 1010 & 7.0 & 0.2 & abs & $0-1$ & abs & abs & abs & abs \\
\hline
\end{tabular}

1 2). Procalcitonin test, coagulation tests, thyroid hormones and aldosterone - within normal range. Infectious screening, including RPR-test for Treponema Pallidum, HBsAg, anti-HCV and anti-HIV-antibodies, PCR for VEB, CMV, HSV type 1 and 2 was negative. Chest X-Ray was otherwise normal, abdomen and kidney ultrasound unremarkable.

Diagnostic considerations and final diagnosis: At that point nephrologists were invited to see the patient and search for polyuria and hypokalemia cause. Given patients history, presence of acidosis, normal sodium and magnesium levels, normal thyroid hormones level and normal blood pressure, the most often causes of hypokalemia, like excessive sweating, diarrhea, starvation, milk-alkali syndrome, drug-induced potassium losses, hyperaldosteronism, hyperreninemia, Bartter,
Gitelman's and Liddle syndromes and thyrotoxicosis were ruled out. Pseudohypokalemia was also ruled out as leukocytosis was not that prominent and patient had multiple symptoms of true hypokalemia.

Additional history taking revealed that since adolescence the patient used to consume Coca-Cola about 2 L per day and more every day, without any signs and symptoms but thirst and stools 2-3 times a day. At the onset of present illness her thirst increased and she consumed as much as 5 L per day, mostly CocaCola and water.

That led us to the diagnosis of hypokalemia with myopathy; rhabdomyolysos, atrial fibrillation and hypokalemic nephropathy due to excessive Coca-Cola consumption. We presume that she 
had chronic hypokalemia with asymptomatic myopathy for a long time. Physical exercise triggered rhabdolyolysis with fever, inflammatory response and exaggeration of hypokalemia with typical symptoms like paralysis, arrhythmia and polyuria. She most probably also had hypokalemic nephropathy long before, as the duration of Coca-Cola consumption exceed 20 years.

Further treatment and follow-up: Patient was discharged from ICU and treated with oral potassium formulations in nephrology unit for next 8 days. Her neurological symptoms completely resolved, serum potassium was normal as well as enzymes and blood count, but her urinalysis still showed low specific gravity and mild proteinuria (Tables 1-3). She was discharged after totally 2 weeks of hospital stay and advised to avoid Coca-Cola consumption. Three month later at out-patient consult she is doing well, no complains, taking no medications. Her serum potassium is quite normal; however her urine specific gravity is still low - 1012-1013.

\section{Conclusion}

in cases of symptomatic unexplained hypokalemia rare causes like excessive Coca-Cola consumption should be considered hypokalemic nephropathy is unusual finding in such cases; its presence may confirm prolonged hypokalemia even though it was not diagnosed previously.

\section{References}

1. Rubin ME. Water excrtion in potassium-deficient man. J J Clin Invest. 1961;40(12):2215-2224.

2. Berl T, Linas S, Aisenbrey G, Anderson R. On the mechanism of polyuria in potassium depletion. J Clin Invest. 1977;60(3):620-625.

3. Torres V, Young W, Offord K, Hattery, RR. Association of hypokalemia, aldosteronism, and renal cysts. N Engl J Med. 1990;322-345.

4. Menahem S, Perry G, Dowling J, Thomson N. Hypokalaemia-induced acute renal failure. Nephrol Dial Transplant. 1999;14:2216.

5. Siscovick DS, Raghunathan TE, Psaty BM, Koepsell TD, Wicklund KG, Lin X, Cobb L, et al. Diuretic therapy for hypertension and the risk of primary cardiac arrest. N Engl J Med. 1994;330(26):1852-7.
6. Singhal P, Abramovici M, Venkatestan J, Hypokalemia, rhabdomyolysis. Miner Electrolyte Metab, et al. 1991;17,335-339.

7. West M, Marsden P, Richardson R. New clinical approach to evaluate disorders of potassium excretion. Miner Electrolyte Metab. 1986;12(4):234-8.

8. Greenfeld D, Mickley D, Quinlan D, Roloff P. Hypokalemia in outpatients with eating disorders. Am J Psychiatry. 1995;152(1):60-3.

9. Shafi T, Appel LJ, Miller ER, Klag MJ, Parekh RS. Changes in Serum Potassium Mediate Thiazide-Induced Diabetes. Hypertension. 2008;52(6):1022-9. doi: 10.1161/HYPERTENSIONAHA.108.119438.

10. Bichet DG, Fujiwara TM. Reabsorption of sodium chloride--lessons from the chloride channels. N Engl J Med. 2004;350(13):1281-3.

11. Harvey T. Addison's disease and the regulation of potassium: the role of insulin and aldosterone. Med Hypotheses. 2007;69(5):1120-6.

12. Matsunami K, Imai A, Tamaya T. Hypokalemia in a pregnant woman with long-term heavy cola consumption. Int J Gynaecol Obstet. 1994;44(3):283-4.

13. Rice J, Faunt J. Excessive cola consumption as a cause of hypokalaemic myopathy. Intern Med J, 2001;31, 317-318.

14. Lee H J, Kim W D, Cho S H, Lung E Y, Lee D W, Chang S H, et al. Hypokalaemic paralysis inducedby large amounts of cola consumption. Nephrol. Dial. Transplant. 2007;22(9):2729.

15. Alazami M, Lin S-H, Cheng C-J, Davids M, Halperin M. Unusual causes of hypokalaemia and paralysis. QJM. 2006;99(3):181-92.

16. Kaya H, Gökdemir M, Söğüt Ö, Albayrak L. Hypocalemic Myopathy Due to Excessive Cola Consumption. Tr J Emerg Med. 2012;12(3):137-139. doi: 10.5505/1304.7361.2012.87004.

17. Dubey D, Sawhney A, Sharma A, Dubey D. Paroxysmal paralytic attacks secondary to excessive cola consumption. CM\& Rrapid Release. Clin Med Res. 2014;12(1-2):61-64. doi:10.3121/cmr.2013;1167.

18. Packer CD. Chronic hypokalemia due to excessive cola consumption: a case Report. Cases J. 2008;1:32. doi: 10.1186/1757-1626-1-32.

19. Tsimihodimos V, Kakaidi V, Elisaf M. Cola-induced hypokalaemia: pathophysiological mechanisms and clinical implications. Int J Clin Pract. 2009;63(6):900-2. doi: 10.1111/j.1742-1241.2009.02051.x. 